\title{
Democratising in situ structural biology: when a field becomes a tool
}

Alex de Marco ${ }^{1}$, Sergey Gorelick ${ }^{1}$, Cyntia Taveneau ${ }^{2}$, Xuan Cheng ${ }^{2}$, David Dierickx ${ }^{2}$ and Genevieve Buckley $^{2}$

${ }^{1}$ Monash University, Clayton, Australia, ${ }^{2}$ Monash University, United States

Today, cryo-electron microscopy (cryo-EM) can deliver incredible results in both structural and cell biology. Despite the great potential that this technique has widely demonstrated the major bottleneck, across the entire range of applications, is currently at the level of sample preparation.

Over a decade ago, cryo-Focused Ion Beam (FIB) milling was introduced to target specific regions in a cell using Cryo-Electron Tomography (cryo-ET). Unfortunately, the throughput of this procedure for a long time was limited to 6-8 cells/day. My team has automated the cryo-FIB milling procedure and increased the throughput by $\sim 10$-fold, showing the preparation of up to 5 cells/hour [1]. Further, in order to simplify and increase the efficiency of cryo-FIB preparation, we developed the Photon-Ion-Electron microscope (PIE-scope), an integrated cryo-LM and cryo-FIB [2-3]. The integration of a fluorescence microscope with a cryo-FIB allows the seamless performance of cryo-Correlative microscopy, eliminating the risks of sample contamination while transferring it between imaging modalities. Further, it enables the automation of the correlation procedure. Finally, in collaboration with Thermo Fisher, our team tested and introduced a new category of unconventional Plasma-sources optimised for life science samples. Our results demonstrate that the oxygen beam can double the throughput of FIB/SEM tomography on resin embedded samples [4]. Further, we demonstrated that the use of light atoms beams such as argon is ideal for milling frozen-hydrated samples, and despite being more challenging to use, they provide superior lamella quality [under review]. All algorithms for automated cryo lamella preparation, the mechanical design and the control software for the PIE-scope are freely available on https://github.com/demarcolab.

I will present what can be achieved using an integrated correlative light and FIB/SEM setup, I will discuss the latest bottlenecks we identified in this field and show what are our current efforts to ease the adoption of in situ cryo-ET for labs that have no prior experience or local expertise.

\section{References}

[1]. G Buckley, G Gervinskas, C Taveneau, H Venugopal, JC Whisstock, A de Marco. Automated cryolamella preparation for high-throughput in-situ structural biology. Journal of structural biology 210 (2), 107488

[2]. S Gorelick, G Buckley, G Gervinskas, TK Johnson, A Handley, JC Whisstock, R Pocock, A de Marco. PIE-scope, integrated cryo-correlative light and FIB/SEM microscopy. Elife 8, e45919

[3]. S Gorelick, DA Dierickx, G Buckley, JC Whisstock, A de Marco. Assembly and Imaging Set up of PIE-Scope. BioProtocol 10 (19), e3768 